\title{
Perioperative Results and Risk Factors for In-Hospital Mortality In Patients With Stanford Type A Aortic Dissection Undergoing Sun's Procedure - A Single Center Study
}

\author{
Yuhui Wu, ${ }^{1,2}$ Rui Jiang, ${ }^{3}$ Ping Xu, ${ }^{1}$ Guanrong Wang, ${ }^{3}$ Jianhua Wang, ${ }^{3}$ Sumin Yang ${ }^{1 *}$ \\ ${ }^{1}$ Department of Cardiovascular Surgery, the Affiliated Hospital of QingDao University, No.16 JiangSu Road, QingDao \\ 266003, China; ${ }^{2}$ Department of Cardiology, the Affiliated Cardiovascular Hospital of QingDao University, No. 5 ZhiQuan \\ Road, QingDao 266071, China; ${ }^{3}$ Department of Operation Room, the Affiliated Hospital of QingDao University, No.16 \\ JiangSu Road, QingDao 266003, China
}

\section{ABSTRACT}

Background: The study was to analyze the therapeutic effect and risk factors of in-hospital mortality in patients with acute Stanford type A aortic dissection operated by Sun's procedure.

Methods: From Jan. 2010 to March 2016, 72 patients whose data was fully accessible underwent Sun's procedure in our hospital due to acute Stanford type A aortic dissection. Patients were divided into the survival group and the death group, and the risk factors for in-hospital mortality were collected and analyzed.

Results: All 72 patients were diagnosed as acute Stanford type A aortic dissection by CT angiography in which the ascending aorta, aortic arch and descending aorta were involved; these patients were operated by Sun's procedure. The operation of proximal aorta included 39 Bentall procedure, one David surgery, and 32 ascending aorta replacement. The in-hospital mortality rate was $19.4 \%$ (14 patients). Studies showed the risk factors for the in-hospital mortality included the body mass index, cardiopulmonary bypass time, operation time, intraoperative transfusion of red blood cells and plasma volume, and the total perioperative transfusion of red blood cells, plasma and cryoprecipitate volume. Independent risk factors included the body mass index and cardiopulmonary bypass time.

Conclusion: Acute Stanford type A aortic dissection is a severe, complex disease with high in-hospital mortality, though the Sun's procedure is an effective surgical approach in treating this kind of disease in some center. Body mass index and cardiopulmonary bypass time are independent risk factors for in-hospital mortality.

\section{BACKGROUND}

Stanford type A aortic dissection (TAAD) is an acute, severe, and life-threatening disease, and usually has a poor

Received September 13, 2017; accepted May 16, 2018.

Correspondence: Sumin Yang, the Affiliated Hospital of QingDao University, No.16 7iangSu Road, QingDao 266003, China; +86-532-82911011 (e-mail: suminyangcn@163.com). result if it is not treated with surgical intervention. The mortality of TAAD increased by $1 \%$ per hour within the first 48 hours after the initial onset, and can reach about $70 \%$ within one week, even $90 \%$ within two weeks. Usually the dissection is considered in the acute stage if the time from the onset of the symptoms to surgery is 14 days or less. The emergency of acute type A aortic dissections is defined by the high propensity to quickly develop severe complications including aortic rupture, severe aortic regurgitation, pericardial tamponade, and cerebral and coronary malperfusion. In recent years, the diagnostic rate of aortic dissection increased significantly due to the knowledge to aortic dissection and the popularization of CT angiography (CTA). Surgery is an effective method to rescue the life of patients with TAAD [Hagan 2000], and in the early years, the ascending aorta or partial aortic arch replacement was usually performed. With the knowledge of aortic dissection and the improvement of surgical technique, Kato et al. [Kato 1996] first reported that they used stent "trunk" to treat TAAD, many surgeons adopt this procedure [Mizuno 2002]. Sun et al [Sun 2008; Sun 2009] introduced the procedure of total arch replacement with stented elephant trunk implantation (Sun's procedure) for TAAD involved in the aortic arch and descending aorta and had achieved good clinical results. His team reported that the in-hospital mortality was $4.73 \%$, and the re-operation rate was 2.34\% [Sun 2013; Ma 2013; Ma 2013]. By analyzing the patients treated by the Sun's procedure retrospectively in our center, we want to explore the therapeutic effect of the procedure and the risk factors for in-hospital mortality.

\section{METHODS}

From Jan. 2010 to March 2016, 72 consecutive selected patients with TAAD involved in the aortic arch and descending aorta diagnosed by preoperative CTA underwent Sun's procedure in our hospital. The clinical data is shown in Table 1. In order to ensure the integrity and accuracy of the data, all examinations were completed in our center. Sixty-three patients were male, and nine patients were female, with an average age $(47.6 \pm 9.6)$ years old, and the average body mass index $(\mathrm{BMI})(26.0 \pm 3.8) \mathrm{kg} / \mathrm{m}^{2}$. Fifty-three patients had hypertension. Sudden severe chest pain was the initial 
Table 1. Preoperative patient characteristics

\begin{tabular}{lc}
\hline Characteristics & Total $(\mathrm{N}=72)$ \\
\hline Age (years), $\pm \mathrm{SD}$ & $47.6 \pm 9.6$ \\
Gender, male/female (\%) & $63(87.5 \%) / 9(12.5 \%)$ \\
BMI $\left(\mathrm{kg} / \mathrm{m}^{2}\right), \mathrm{M} \pm \mathrm{SD}$ & $26.0 \pm 3.8$ \\
Admission to operation & \\
$\quad$ Time(days), $\mathrm{M} \pm \mathrm{SD}$ & $1.6 \pm 4.4$ \\
Onset of symptoms to operation & \\
$\quad$ Time (days), $\mathrm{M} \pm \mathrm{SD}$ & $4.3 \pm 6.6$ \\
Emergent operation $(\%)$ & $61(84.7 \%)$ \\
Hypertension (\%) & $53(73.6 \%)$ \\
Blood urea nitrogen $(\mathrm{mmol} / \mathrm{L}), \mathrm{M} \pm \mathrm{SD}$ & $6.6 \pm 2.4$ \\
Creatinine $(\mu \mathrm{mol} / \mathrm{L}), \mathrm{M} \pm \mathrm{SD}$ & $86.9 \pm 31.9$ \\
White blood cell count $\left(\times 10^{\%} / \mathrm{L}\right), \mathrm{M} \pm \mathrm{SD}$ & $13.0 \pm 4.9$ \\
LVEDD (cm), $\mathrm{M} \pm \mathrm{SD}$ & $4.9 \pm 0.6$ \\
LVEF (\%), $\mathrm{M} \pm \mathrm{SD}$ & $63.5 \pm 5.3$ \\
\hline
\end{tabular}

BMI=body mass index, LVEDD= left ventricular end-diastolic dimension, $L V E F=$ left ventricular ejection fraction

symptom in 69 cases, whereas abdominal pain was the initial symptom in three cases. The indications for total arch replacement were the following conditions: (1) a primary tear in the transverse arch or the proximal descending aorta, and (2) serious involvement of the arch vessels. In this study, the primary tear of the aortic dissection was mainly in the arch or proximal descending aorta, besides 10 patients in the ascending aorta near to the arch in which the arch vessels were seriously involved. Most of the patients (61 patients, $84.7 \%$ ) were operated on emergently or urgently after admission. The study protocol was approved by the Committee for the Protection of Human Subjects at the Affiliated Hospital of Medical College, Qingdao University. Informed consent was obtained from each patient involved in this study.

\section{SURGICAL PROCEDURE}

The left radial artery and double dorsalis pedis artery were punctured to monitor blood pressure. Anesthesia was induced and maintained, according to accepted, standard procedures. A near-infrared spectroscopy sensor was placed to assess the brain perfusion. All operations were performed through a median sternotomy. The right axillary artery was routinely exposed for the arterial perfusion, and the right atrium or the superior and inferior veva cava were cannulated for venous drainage. After heparinization, the cardiopulmonary bypass (CPB) was established. The left ventricle was vented through the right superior pulmonary vein. Routinely, the artery line was bifurcated; one for the right axillary artery perfusion and the other for antegrade perfusion through one limb of the four-branch prosthetic graft. Systemic cooling was initiated when the $\mathrm{CPB}$ started. The ascending aorta was clamped and longitudinally opened, and the cold-blood cardioplegic solution was directly infused into the left and right coronary artery separately, and repeated every 20 minutes to 30 minutes for the myocardial protection. During the cooling process, the aortic root surgery, including the ascending aorta replacement or David or Bentall surgery, was performed depending on the aortic root lesions. When the surgery was finished, we sutured the bleeding in the anastomotic site found by directly infusing the cold-blood cardioplegic solution through the aortic root. When the nasopharyngeal temperature reached $17^{\circ} \mathrm{C}$ to $26^{\circ} \mathrm{C}$, and the rectal temperature reached $19^{\circ} \mathrm{C}$ to $29^{\circ} \mathrm{C}$, we clamped the brachiocephalic artery, left common carotid artery and left subclavian artery sequentially, the deep hypothermic circulatory arrest (DHCA) and selective cerebral perfusion (SCP) through the right axillary artery $(5 \mathrm{ml} / \mathrm{kg} \sim 10 \mathrm{ml} / \mathrm{kg}$ ) were initiated. The head was packed in ice. We then removed the aortic forceps, opened the aortic arch longitudinally, and sought the intimal tear or entry of the dissection. The aortic arch was transected between the left common carotid artery and left subclavian artery. We released the stented elephant graft (MicroPort Medical Co Ltd, Shanghai, China) firstly in the descending aorta, and the size was determined by surgeons according to the preoperative CTA. The residual of the left subclavian artery in the arch was sutured directly. After the anastomosis of the three parts including the stented graft, descending aorta and four-branch prosthetic graft, the lower body was immediately reperfused by perfusing one limb of the four-branch prosthetic graft with half of the normal flow. The brain was perfused bilaterally when the anastomosis to the left common carotid artery was finished. After that, the $\mathrm{CPB}$ gradually resumed to its normal flow and rewarming began, and we checked and sutured the bleeding on the distal anastomosis. The heart was resuscitated when we finished the anastomosis between the proximal aortic arch and the ascending aorta. Then, the brachiocephalic artery and left subclavian artery were anastomosed with the corresponding branch of the four-branch prosthetic graft separately. Finally, we built a drainage to the right atrium using the residual arterial wall or the pericardium or biological patch around the artificial graft.

\section{STATISTICAL ANALYSIS}

The statistical analyses were finished with the SPSS 19.0 software. Continuous data was expressed with mean \pm standard deviation, while categorical data was expressed as ratio or percentage. Differences between the groups were tested with Fisher's exact test for categorical variables and t-test for continuous variables. The variables which were significantly $(P<.05)$ associated with the in-hospital mortality analyzed by the univariate analysis were analyzed by the multivariate logistic regression analysis to identify the independent factors. A $P$-value of less than 0.05 indicated a significant difference.

\section{RESULTS}

All 72 patients with TAAD were operated by Sun's procedure. There were 63 males and nine females in this group, 
Table 2. Univariate analysis for perioperative risk factors of the in-hospital mortality in patients underwent Sun's surgery

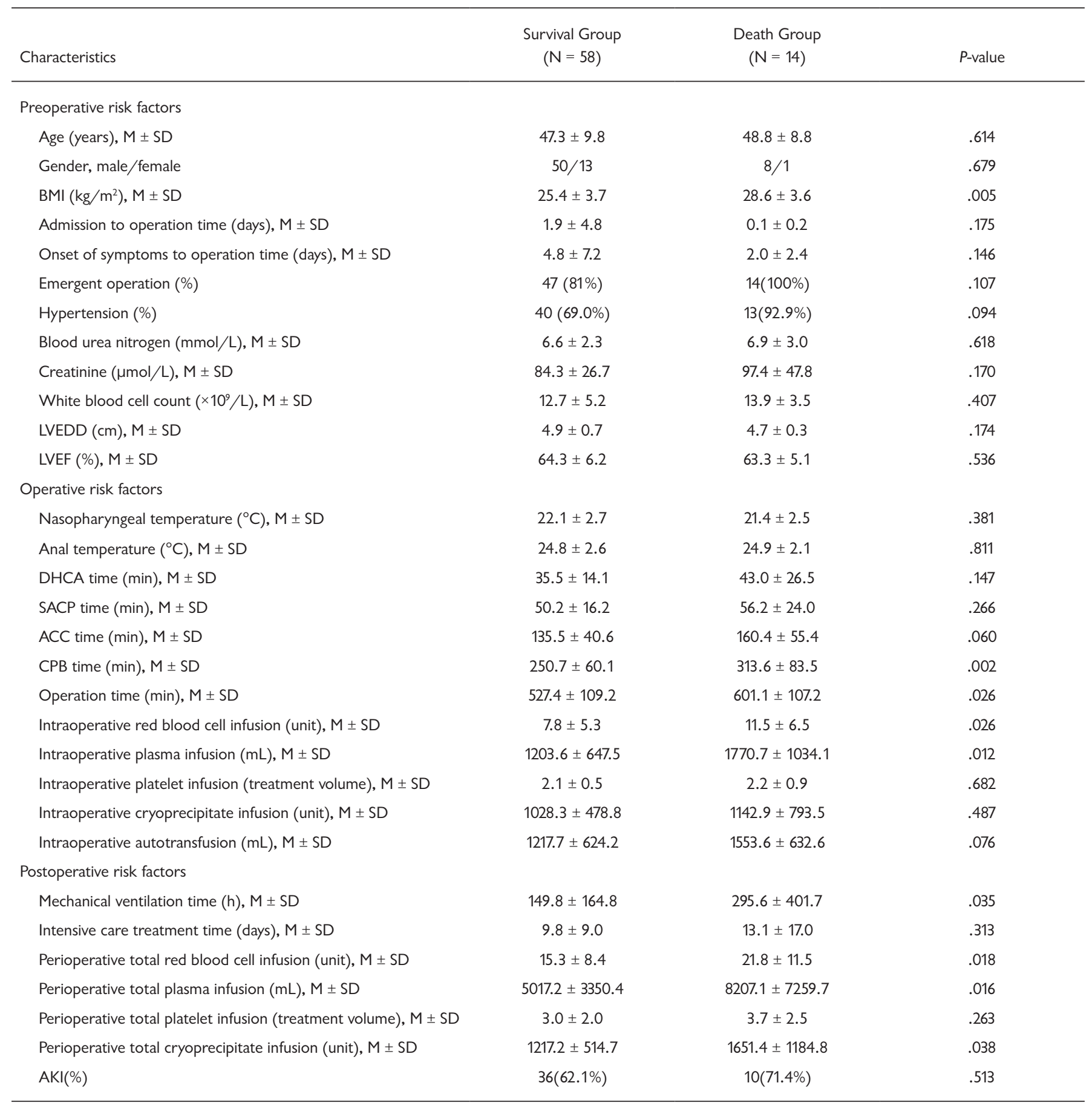

$\mathrm{DHCA}=$ deep hypothermic circulatory arrest, $\mathrm{SACP}=$ selective antegrade cerebral perfusion, $\mathrm{ACC}=$ aortic cross clamp, $\mathrm{CPB}=$ cardiopulmonary bypass, $\mathrm{AKI}=$ acute kidney injury

with the mean age of $(47.6 \pm 9.6)$ years old. The proximal aortic surgery included Bentall procedure in 39 cases, David surgery in one case, and ascending aorta replacement in 32 cases. The average time from the onset of the symptom to operation was $(4.3 \pm 6.6)$ days, and the average time from admission to operation was $(1.6 \pm 4.4)$ days. The ratio of the emergent or urgent operation was $84.7 \%$ (61patients). The incidence of postoperative acute kidney injury (AKI) was $63.9 \%$ (46 cases). Fourteen patients died after the operation, the in-hospital mortality was $19.4 \%$. Postoperative ICU stay 
Table 3. Multivariate logistic regression analysis model for the in-hospital mortality

\begin{tabular}{|c|c|c|c|c|c|c|c|c|}
\hline & B & S.E. & Wald & $d f$ & Sig & $\operatorname{Exp}(B)$ & $95 \%$ C.I. for $\operatorname{Exp}(B)$ & $\begin{array}{c}\text { Lower Upper } \\
\text { step 1a }\end{array}$ \\
\hline BMI & 0.281 & 0.127 & 4.911 & 1 & 0.027 & 1.324 & 1.033 & 1.698 \\
\hline CPB time & 0.026 & 0.012 & 4.68 & 1 & 0.031 & 1.027 & 1.002 & 1.051 \\
\hline Intraoperative plasma infusion & 0.001 & 0.001 & 2.788 & 1 & 0.096 & 1.001 & 1 & 1.003 \\
\hline Perioperative total red blood cell infusion & -0.125 & 0.095 & 1.737 & 1 & 0.188 & 0.883 & 0.733 & 1.063 \\
\hline Perioperative total plasma infusion & 0 & 0 & 0.088 & 1 & 0.766 & 1 & 1 & 1.001 \\
\hline Perioperative total cryoprecipitate infusion & 0.001 & 0.001 & 0.944 & 1 & 0.331 & 1.001 & 0.999 & 1.002 \\
\hline
\end{tabular}

$\mathrm{BMI}=$ body mass index, $\mathrm{CPB}=$ cardiopulmonary bypass

time was $(10.4+10.9)$ days, and the average hospitalization time was $(22.0+15.0)$ days. Univariate analysis of the perioperative characteristics showed the following variables were found to have significant differences between the two groups, such as BMI, CPB time, operation time, mechanical ventilation time, amount of red blood cells and plasma infusion during operation, and the total amount of perioperative red blood cells, plasma, and cryoprecipitate infusion (Table 2). As shown in Table 3, BMI and CPB time were the independent risk factors for in-hospital mortality confirmed by the multivariate logistic regression analysis in TAAD in our center. During the follow-up, six patients died of the multiple organ dysfunction, nine patients were lost. We found that the true lumen of the descending aorta expanded significantly in the rest of 43 patients, and the false lumen became thrombosis in 40 of them. Only three patients were found to have an obvious dissection in the distal of the stented graft, but these patients had unobvious symptoms and the dilation of the descending aorta was not significant by controlling the blood pressure with medicine during the follow-up, so no re-operation was performed.

\section{DISCUSSION}

In recent years, along with the acknowledgement of aortic dissection and the popularity of CTA, the diagnostic accuracy of aortic dissection increased annually in our country. The most common symptoms for Stanford type A aortic dissection were sudden severe chest pain, and the reported ratio was $74 \%$ to $90 \%$. While in our hospital, the ratio was $95.8 \%$. TAAD is an urgent, serious disease with high mortality, reaching nearly $50 \%$ in the first 48 hours. Prognosis will be extremely poor if it's not treated with surgery, which has been acknowledged as an effective way in treating TAAD. The ascending aorta or partial aortic arch replacement was performed in the early years, but studies have shown this kind of surgery would leave false lumen or cause bleeding difficult to control in the operation and had a high incidence of postoperative long-term aortic rupture or aneurysm [Liu 2006]. Then Kato et al reported that they obtained better therapeutic effect by using stent "trunk" to treat TAAD. In our country, professor Sun [Sun 2008; Sun 2009] recommended the total arch replacement with stented elephant trunk implantation (Sun's procedure) for TAAD involved in the aortic arch and descending aorta. Sun's procedure can enlarge the resection of the aortic dissection. The self-expanding stented trunk plays an important role in closing the inlet of the dissection, enlarging the true lumen of the descending aorta, reconstructing the aortic wall, and it also reduced the incidence of bleeding in the distal anastomosis caused by the reverse blood flow from the false lumen. Furthermore, a stented elephant trunk can offer a suture site in the operation of thoracoabdominal aortic replacement in future. However, Sun's procedure is a complex operation with high mortality, which requires DHCA, SCP, long CPB time and operation time compared with the general cardiac surgery, accompanied by severe trauma, multiple vascular anastomosis, bleeding, more blood transfusion, and so on. Despite the improvements in surgical techniques and postoperative care for acute Stanford type A aortic dissection, the registry data published in 2015 suggests a persistently high operative mortality of $15 \%$ to $20 \%$ [Pape 2015; Czerny 2015], even some studies reported the surgical mortality ranged from $17 \%$ to 26\% [Conzelmann 2016; Knipp 2007], and TAAD continues to represent a great challenge for aortic surgeons. In recent years, 72 patients underwent Sun's procedure in our center and the clinical outcome was satisfactory, 14 cases died in the hospital after operation, and the mortality was $19.4 \%$, which was consistent with the above reports. Though the mortality seemed higher compared with some studies [Zierer 
2017; Lu 2016], we thought the high mortality in our study might be associated with the following reasons by analyzing the above studies. On one hand, the patients in our study all were operated by Sun's procedure, except the ascending aorta or partial aortic arch replacement. On the other hand, from Table 1, we can find most of the operations were emergent or urgent $(84.7 \%$ ) because the patients were in an unstable state, including persistent chest pain, uncontrollable hypertension, and so on. The average time from the onset of symptoms to operation and from admission to operation was $(4.3 \pm 6.6)$ days and $(1.6 \pm 4.4)$ days, most of the patients were operated within 10 hours following admission. The risk factors for inhospital mortality in our center were BMI, CPB time, operation time, mechanical ventilation time, the amount of intraoperative red blood cell and plasma infusion, and the amount of perioperative red blood cells, plasma, and cryoprecipitate infusion. BMI and CPB time were the independent risk factors. Studies have shown that obesity $\left(\mathrm{BMI} \geq 25 \mathrm{~kg} / \mathrm{m}^{2}\right)$ is the independent risk factor of hypoxemia [Howard 2014], which may raise the inflammation level, significantly delay the extubation time, and prolong the length of stay in ICU [Aizawa 2013; McCallister 2009]. Our study shows that the patients in the death group have big BMI, long mechanical ventilation time and ICU hospitalization time (Table 2). Patients with a high BMI need a longer time in the cooling and rewarming process, so the $\mathrm{CPB}$ time for these patients is longer. Patients with complex aortic surgery usually need a large amount of blood transfusion due to perioperative blood loss. The bleeding in the distal anastomosis is the most common cause of death in the aortic dissection operation. Sun's procedure needs to implant a lot of artificial vascular graft [Di 2013], which requires much more time to complete the vascular anastomosis compared with the simple ascending aorta or partial aortic arch replacement. If the bleeding in the operation was difficult to deal with, we needed much more time to control it, and sometimes we had to re-operate under the process of CPB or even DHCA again, thus all of these processes increased the CPB and total operation time. Studies have shown that prolonged $\mathrm{CPB}$ time is the risk factor of the postoperative complications such as prolonged mechanical ventilation after cardiovascular surgery, renal failure and gastrointestinal complications, and even death [Natarajan 2006; Geissler 2006; Pedersen 2007]. The inflammation caused by factors, such as the contact of blood with the surface of conduit in $\mathrm{CPB}$, the perfusion pattern, large dose of heparin anticoagulation, deep hypothermia and long $\mathrm{CPB}$ time, may have an inevitable effect on blood coagulation function [Yavari 2009]. Study showed that prolonged CPB time might severely damage the function of the platelet, which in turn could damage the coagulation system [Yeh 2005]. These factors can increase the risk of re-operation and the amount of perioperative blood transfusion. In recent years, many studies show that perioperative blood transfusion is not only related to perioperative complications of the cardiac surgery, but also affects the patient's recent and long-term survival [Kuduvalli 2005; Surgenor 2009], and blood transfusion is the independent risk factor for mortality after cardiac surgery [Surgenor 2009; Paone 2014; van Straten 2010]. Stanworth et al found the mortality was $9 \%$ in patients who received zero five packed red blood cells (PRBC) units, $22 \%$ in patients receiving six-nine PRBC units and $42 \%$ in patients receiving 10 or more units [Stanworth 2010]. Surgenor et al suggested that the high mortality was related to the infusion of red blood cells (RBCs) intraoperative or postoperative during the cardiac surgery, and with a $16 \%$ higher risk of death in patients who received one or two units of RBCs [Surgenor 2009].

\section{CONCLUSIONS}

Our data showed that the risk factors for in-hospital mortality of the patients operated by the Sun's procedure were BMI, CPB time, operation time, mechanical ventilation time, the amount of intraoperative red blood cell and plasma infusion, and the amount of perioperative red blood cells, plasma, and cryoprecipitate infusion. $\mathrm{BMI}$ and $\mathrm{CPB}$ time were independent risk factors. Acute Stanford type A aortic dissection is still a severe, complex disease with high in-hospital mortality in patients operated by Sun's procedure in the acute stage, though the procedure is an effective method to treat this kind of disease in some centers.

\section{REFERENCES}

Aizawa K, Sakano Y, Ohki S, Saito T, Konishi H, Misawa Y. 2013.Obesity is a risk factor of young onset of acute aortic dissection and postoperative hypoxemia. Kyobu Geka 66(6): 437-444.

Conzelmann LO, Weigang E, Mehlhorn U, et al. 2016. Mortality in patients with acute aortic dissection type A: analysis of pre- and intraoperative risk factors from the German Registry for Acute Aortic Dissection Type A(GERAADA). Eur J Cardiothorac Surg 49(2):e44-52.

Czerny M, Schoenhoff F, Etz C, et al. 2015. The impact of preoperative malperfusion on outcome in acute type A aortic dissection: results from the GERAADA registry. J Am Coll Cardiol 65(24):2628-2635.

Di Eusanio M, Di Bartolomeo R. 2013. The Sun procedure-a new paradigm of treatment in DeBakey type 1 acuteaortic dissection? Ann Cardiothorac Surg 2(5): 629-630.

Geissler HJ, Fischer UM, Grunert S, et al. 2006. Incidence and outcome of gastrointestinal complications after cardiopulmonary bypass. Interact Cardiovasc Thorac Surg 5(3): 239-242.

Hagan PG, Nienaber CA, Isselbacher EM, et al. 2000. The international registry of acute aortic dissection (IRAD): new insights into an old disease. JAMA 283(7): 897-903.

Howard LS, Santhirapala V, Murphy K, et al. 2014. Cardiopulmonary exercise testing demonstrates maintenance of exercise capacity in patients with hypoxemia and pulmonary arteriovenous malformations. Chest 146(3):709-718.

Kato M, Ohnishi K, Kaneko M, et al. 1996. New graft implanting method for thoracic aortic aneurysm or dissection with a stented graft. Circulation 94(Suppl 9):188-193.

Knipp BS, Deeb GM, Prager RL, Williams CY, Upchurch GR Jr, Patel HJ. 2007. A contemporary analysis of outcomes for operative repair of type A aortic dissection in the United States.Surgery142(4):524-8; discussion 528.e1.

Kuduvalli M, Oo AY, Newall N, et al. 2005. Effect of perioperative red blood cell transfusion on 30-day and 1-year mortality following coronary 
artery bypass surgery. Eur J Cardiothorac Surg 27(4): 592-598.

Liu ZG, Sun LZ, Chang Q, et al. 2006. Should the "elephant trunk" be skeletonized? Total arch replacement combined with stented elephant trunk implantation for Stanford type A aortic dissection. J Thorac Cardiovasc Surg 131(1): 107-113.

Lu SY, Yang SG, Lai H, et al. 2016. Open aortic arch reconstruction for acute type A aortic dissection: a single-center experience with 267 consecutive patients. J Cardiothorac Surg 11(1):111.

Ma WG, Zheng J, Dong SB, et al. 2013. Sun's procedure of total arch replacement using a tetrafurcated graft with stented elephant trunk implantation: analysis of early outcome in 398 patients with acute type A aortic dissection. Ann Cardiothorac Surg 2(5): 621.

Ma WG, Zhu JM, Zheng J, et al. 2013. Sun's procedure for complex aortic arch repair: total arch replacement using a tetrafurcate graft with stented elephant trunk implantation. Ann Cardiothorac Surg 2(5): 642.

McCallister JW, Adkins EJ, O'Brien JM Jr. 2009. Obesity and acute lung injury. Clin Chest Med 30(3): 495-508.

Mizuno T, Toyamab M, Tabuchi N, Wu H, Sunamori M. 2002. Stented elephant trunk procedure combined with ascending aorta and arch replacement for acute type A aortic dissection. Eur J Cardiothoracic Surg 22: 504-509.

Natarajan K, Patil S, Lesley N, Ninan B. 2006. Predictors of prolonged mechanical ventilation after on-pump coronary artery bypass grafting. Ann Card Anaesth 9(1): 31-36.

Sun LZ, Ma WG, Zhu JM, et al. 2013. Sun's procedure for chronic type A aortic dissection: total arch replacement using a tetrafurcate graft with stented elephant trunk implantation. Ann Cardiothorac Surg 2(5): 665.

Paone G, Likosky DS, Brewer R, et al. 2014. Transfusion of 1 and 2 units of RBCs is associated with increased morbidity and mortality. Ann Thorac Surg 97(1): 87-93.
Pape LA, Awais M, Woznicki EM, et al. 2015. Presentation, diagnosis, and outcomes of acute aortic dissection: 17-year trends from the international registry of acute aortic dissection. J Am Coll Cardiol 66(4):350-358.

Pedersen KR, Povlsen JV, Christensen S, et al. 2007. Risk factors for acute renal failure requiring dialysis after surgery for congenital heart disease in children. Acta Anaesthesiol Scand 51(10): 1344-1349.

Stanworth SJ, Morris TP, Gaarder C, et al. 2010. Reappraising the concept of massive transfusion in trauma. Crit Care 14(6): R239.

Sun LZ, Qi RD, Chang Q, et al. 2008. Surgery for marfan patients w ith acute type A dissection using a stented elephant trunk procedure. Ann Thorac Surg 86(6): 1821-1825.

Sun LZ, Qi RD, Chang Q, et al. 2009. Surgery for acute type A dissection with the tear in the descending aorta using a stented elephant trunk procedure. Ann Thorac Surg 87(4): 1177-1180.

Surgenor SD, Kramer RS, Olmstead EM, et al. 2009. The association of perioperative red blood cell transfusions and decreased long-term survival after cardiac surgery. Anesth Analg 108(6): 1741-1746.

van Straten AH, Bekker MW, Soliman Hamad MA, et al. 2010. Transfusion of red blood cells:the impact on short-term and long-term survival after coronary artery bypass grafting, a ten-year follow-up. Interact Cardiovasc Thorac Surg 10(1): 37-42.

Yavari M, Becker RC. 2009. Coagulation and fibrinolytic protein kinetics in cardiopulmonary bypass. J Thromb Thrombolysis 27(1): 95-104.

Yeh T Jr, Kavarana MN. 2005. Cardiopulmonary bypass and the coagulation system[J]. Prog Pediatr Cardiol 21(1): 87-115.

Zierer A, El-Sayed Ahmad A, Papadopoulos N, Detho F, Risteski P, Moritz A et al. 2017. Fifteen years of surgery for acute type A aortic dissection in moderate-to-mild systemic hypothermia. Eur J Cardiothorac Surg 51(1):97. 